\title{
High-molecular-weight polyethylene glycol inhibits myocardial ischemia-reperfusion injury in vivo
}

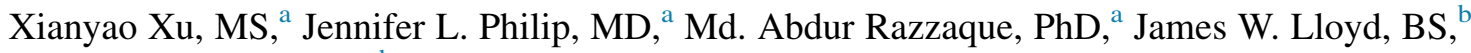 \\ Charlie M. Muller, MD, ${ }^{b}$ and Shahab A. Akhter, MD ${ }^{a}$
}

\begin{abstract}
Objectives: Cardiac ischemia-reperfusion (I-R) injury remains a significant problem as there are no therapies available to minimize the cell death that can lead to impaired function and heart failure. We have shown that high-molecular-weight polyethylene glycol (PEG) (15-20 kD) can protect cardiac myocytes in vitro from hypoxia-reoxygenation injury. In this study, we investigated the potential protective effects of PEG in vivo.
\end{abstract}

\begin{abstract}
Methods: Adult rats underwent left anterior descending artery occlusion for 60 minutes followed by 48 hours or 4 weeks of reperfusion. One milliliter of $10 \%$ PEG solution or phosphate-buffered saline (PBS) control ( $\mathrm{n}=10$ per group) was administered intravenously (IV) immediately before reperfusion.
\end{abstract}

Results: Fluorescein-labeled PEG was robustly visualized in the myocardium 1 hour after IV delivery. The PEG group had significant recovery of left ventricular ejection fraction at 4 weeks versus a $25 \%$ decline in the PBS group $(P<.01)$. There was $50 \%$ less LV fibrosis in the PEG group versus PBS with smaller peri-infarct and remote territory fibrosis $(P<.01)$. Cell survival signaling was upregulated in the PEG group with increased Akt (3-fold, $P<.01$ ) and ERK (4-fold, $P<.05$ ) phosphorylation compared to PBS controls at 48 hours. PEG also inhibited apoptosis as measured by TUNEL-positive nuclei $(56 \%$ decrease, $P<.02)$ and caspase 3 activity (55\% decrease, $P<.05)$.

Conclusions: High-molecular-weight PEG appears to have a significant protective effect from I-R injury in the heart when administered IV immediately before reperfusion. This may have important clinical translation in the setting of acute coronary revascularization and myocardial protection in cardiac surgery. (J Thorac Cardiovasc Surg 2015;149:588-93)

See related commentary on pages 594-5.

We have previously investigated the efficacy of highmolecular-weight (15-20 $\mathrm{kD})$ polyethylene glycol (PEG) as a therapeutic agent to inhibit apoptotic cell death in isolated cardiac myocytes subjected to hypoxiareoxygenation (H-R) injury. ${ }^{1}$ Pretreatment of neonatal rat ventricular myocytes with a 5\% PEG solution led to a 3 -fold decline in apoptosis after $\mathrm{H}-\mathrm{R}$ relative to untreated controls. This was associated with a significant decline in caspase 3 activity and reactive oxygen species (ROS) generation and led to upregulation of prosurvival signaling

From the Division of Cardiothoracic Surgery, ${ }^{\text {a }}$ Department of Surgery, University of Wisconsin School of Medicine and Public Health, Madison, Wis; and the University of Chicago Pritzker School of Medicine, ${ }^{\mathrm{b}}$ Chicago, Ill.

This work was supported, in part, by a Howard Hughes Medical Institute Medical Research Fellowship (to J.L.P.) and the National Institutes of Health (HL107949 to S.A.A).

Disclosures: Authors have to disclose with regard to commercial support.

Received for publication Aug 15, 2014; revisions received Oct 7, 2014; accepted for publication Oct 10, 2014; available ahead of print Nov 11, 2014.

Address for reprints: Shahab A. Akhter, MD, Division of Cardiothoracic Surgery, University of Wisconsin School of Medicine and Public Health, 600 Highland Ave, CSC H4/340, Madison, WI 53792-3236 (E-mail: akhter@surgery.wisc.edu). $0022-5223 / \$ 36.00$

Copyright (c) 2015 by The American Association for Thoracic Surgery

http://dx.doi.org/10.1016/j.jtcvs.2014.10.074 including serine/threonine kinase Akt, extracellular signal-regulated kinase (ERK), and glycogen synthase kinase 3 beta (GSK-3 $\beta$ ) phosphorylation. Sarcolemmal membrane lipid-raft architecture was also preserved, consistent with membrane stabilization, a known protective effect of PEG 15-20 from previous studies in intestinal epithelial cells. ${ }^{2}$ PEG has demonstrated membrane-protective effects in a variety of cells and organs against various insults. PEGs have been shown to decrease ROS generation and lipid peroxidation in these injury models. Because PEGs do not scavenge superoxide anion or inhibit xanthine oxidase, ${ }^{3}$ it is thought that these polymers inhibit or reduce oxidative stress through preservation or restoration of membrane integrity.

The primary goal of this study is to translate our previous in vitro findings of the protective effects of PEG in cardiac myocytes to a small animal in vivo model of acute ischemia-reperfusion (I-R) injury. We used an adult rat model of left anterior descending coronary artery occlusion for 1 hour followed by reperfusion for 48 hours or 4 weeks. To provide more clinical relevance, the PEG solution was delivered intravenously just before reperfusion. The subsequent studies include measurement of left ventricular (LV) function and dimensions, myocardial fibrosis, apoptotic signaling, prosurvival signaling, and overall survival. 

Abbreviations and Acronyms
eNOS = endothelial nitric oxide synthase
ERK = extracellular signal-regulated kinase
GSK-3 $\beta=$ glycogen synthase kinase 3 beta
$\mathrm{H}-\mathrm{R} \quad=$ hypoxia-reoxygenation
$\mathrm{I}-\mathrm{R} \quad=$ ischemia-reperfusion
$\mathrm{LAD}=$ left anterior descending artery
$\mathrm{LV} \quad=$ left ventricular
PBS = phosphate-buffered saline
PEG = polyethylene glycol
ROS $=$ reactive oxygen species

\section{MATERIALS AND METHODS}

High-molecular-weight PEG (15,000-20,000 Da), referred to as PEG 15-20, was purchased from Sigma (St Louis, Mo).

\section{Model of I-R and Delivery of PEG}

All animal studies were approved by the Institutional Animal Care and Use Committee at the University of Wisconsin School of Medicine and Public Health and the University of Chicago Biological Sciences Division. Adult male Sprague-Dawley rats weighing 300 to $350 \mathrm{~g}$ were purchased from Charles Rivers Laboratories (Wilmington, Mass). The animals underwent left thoracotomy and left anterior descending artery (LAD) occlusion at the junction of the proximal and midthird of the vessel for 60 minutes followed by either 48 hours or 4 weeks of reperfusion. LAD occlusion led to a significantly darker color change of the anterior $\mathrm{LV}$, which returned to the baseline color after reperfusion. This was observed routinely in all experiments. One milliliter of $10 \%$ PEG solution or saline control was administered via the internal jugular vein just before reperfusion. For the 48-hour studies, $\mathrm{n}=5$ in both the PEG and saline control (phosphate-buffered saline [PBS]) groups. For the 4-week studies, $\mathrm{n}=11$ in the PEG group and $\mathrm{n}=14$ in the PBS group.

\section{TUNEL Staining}

Using the TUNEL (terminal deoxynucleotidyl transferase [TdT]mediated dUTP nick-end labeling) method, cardiac sections were stained for the detection of fragmented DNA, which is indicative of apoptotic cells, according to the manufacturer's protocol (Chemicon International, Temecula, Calif). The number of TUNEL-positive cells was also counted in 5 different fields.

\section{Histochemistry}

Select heart tissues were perfused and fixed in $10 \%$ buffered formalin overnight at $4{ }^{\circ} \mathrm{C}$ for paraffin embedding. Tissues were then sectioned at $5 \mu \mathrm{m}$, placed on slides and heated at $60^{\circ} \mathrm{C}$ overnight, and deparaffinized in xylene. The sections were then rehydrated in an ethanol series and stained with Masson's Trichrome kit (Sigma), or Picro-Sirius Red (Abcam, Eugene, Ore). For Sirius Red staining, nuclei were stained for 1 minute with Weigert's hematoxylin, washed, and then stained for 1 hour in $0.1 \%$ Sirius Red stain (Direct Red 80, Sigma) in saturated picric acid. Slides were washed twice in acidified water ( $5 \%$ acetic acid), dehydrated in an ethanol series, and cleared with xylene. Sections were cover-slipped with Permount (Sigma). Images were collected using bright field microscopy at 40 to $100 \times$ magnification.

\section{Protein Immunoblotting}

LV tissues were lysed in buffer containing $25 \mathrm{mM}$ 4-(2-hydroxyethyl)-1piperazineethanesulfonic acid (HEPES), $1 \mathrm{mM}$ ethylenediaminetetraacetic acid (EDTA), $125 \mathrm{mM} \mathrm{NaCl}, 0.5 \mathrm{mM} \mathrm{NaF}, 0.25 \%$ Nonidet P-40, $5 \%$ glycerol (pH 6.8), $10 \mu \mathrm{g} / \mathrm{mL}$ leupeptin, $20 \mu \mathrm{g} / \mathrm{mL}$ aprotinin, and $1 \mathrm{mM}$ phenyl-methylsulfonyl fluoride. Equal amounts of protein for each sample were separated by sodium dodecyl sulfate polyacrylamide gel electrophoresis, transferred onto a nitrocellulose membrane, and immunoblotted. Chemiluminescence detection was performed using the ECL reagent (Pierce). Antibodies against phospho Ser473 residues of Akt (1:1000), Ser9 residue of GSK-3 $\beta$ (1:1000), Ser1177 residue of endothelial nitric oxide synthase (eNOS, 1:1000), Thr202/Tyr204 residues of ERK1/2 (1:1000), and cleaved caspase 3 (1:1000) were used (Cell Signaling, Beverly, Mass). Blots were stripped and reprobed with total Akt, GSK-3 $\beta$, eNOS, or ERK1/2 antibodies, respectively, to confirm equal protein loading. Band intensity was quantitated using NIH ImageJ software. $\alpha$-Tubulin was used as a loading control.

\section{Statistical Analysis}

All data are expressed as mean \pm standard error of the mean and analyzed using Student $t$ test. Values of $P<.05$ were considered significant.

\section{RESULTS}

\section{Effects of PEG on Myocardial Function and Mortality After I-R Injury}

Preliminary experiments were done to verify that the $10 \%$ PEG solution was being taken up in the myocardium after intravenous delivery via the right internal jugular vein. This was done using fluorescein-labeled PEG and it was robustly visualized in the myocardium (Figure 1). Following 60 minutes of ischemia induced by LAD occlusion and intravenous delivery of PBS (control) just before reperfusion, fractional shortening declined from a baseline of $50 \%$ to $35 \%$ at 1 week, which did not improve by 4 weeks. In contrast, intravenous delivery of PEG just before reperfusion led to a significant recovery of fractional shortening by 4 weeks at $47 \%$ versus $51 \%$ at baseline (Figure 2, A). Similarly, treatment with PEG led to a near normal recovery of LV ejection fraction compared to PBS controls (Figure 2, $B$ ). End-systolic diameter was increased by 1 week after I-R in both groups and continued to increase by 4 weeks in the PBS group (Figure 2,C). PEG treatment led to stabilization of end-systolic diameter by 2 weeks and was significantly smaller compared to the PBS group at 3 and 4 weeks postreperfusion. Mortality after I-R injury occurred within the first 24 hours and there were no immediate operative deaths or late deaths after 1 day. There was a $40 \%$ mortality rate in the PBS group compared to $10 \%$ in the PEG-treated rats (Figure 2, D).

\section{PEG Treatment Inhibits Ventricular Remodeling Following I-R Injury}

LV fibrosis was measured by Picro-Sirius Red and Masson trichrome staining at 4 weeks post-I-R injury induced by 60 minutes of LAD ligation. Baseline fibrotic area is approximately $1 \%$, and this was markedly increased to $7 \%$ by Picro-Sirius Red staining by 4 weeks in the PBS-treated group (Figure 3, $A$ and $B$ ). PEG treatment significantly inhibited the development of ventricular 

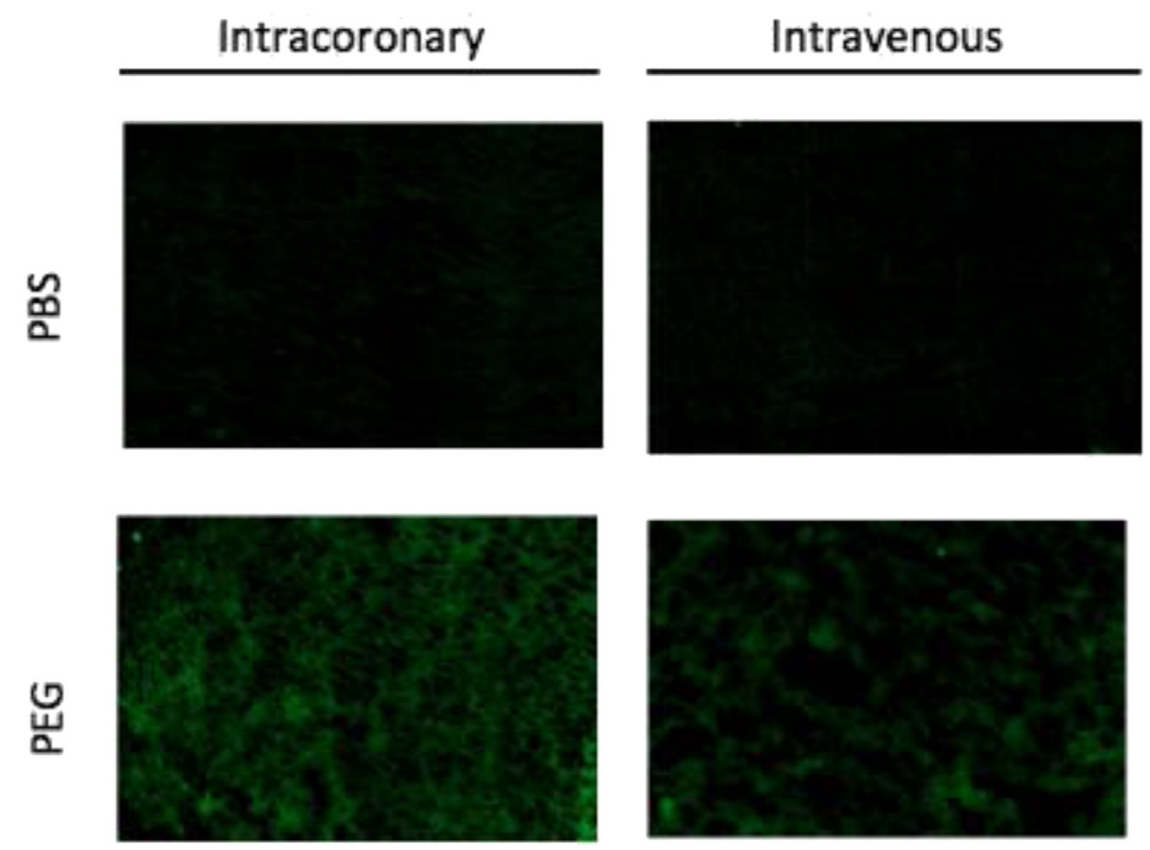

FIGURE 1. PEG 15-20 is present in the myocardium following intracoronary or intravenous delivery. Fluorescein-labeled PEG or PBS (control) was delivered via direct injection into the left ventricular outflow tract following transient aortic crossclamping (intracoronary delivery) or by intravenous delivery via the right internal jugular vein. Both delivery techniques resulted in robust uptake of PEG in the heart, as demonstrated by the green fluorescein labeling. $P E G$, Polyethylene glycol; $P B S$, phosphate-buffered saline.

fibrosis with a fibrotic area of $3 \%$. Fibrosis as measured by trichrome staining showed an increase to nearly $14 \%$ by area in the PBS group versus only $4 \%$ in the PEG group (Figure 3, $C$ and $D$ ).

\section{PEG Inhibits Apoptotic Cell Death and Caspase 3 Activity}

To investigate the potential effects of PEG treatment on myocardial apoptosis in vivo, we measured TUNEL-positive
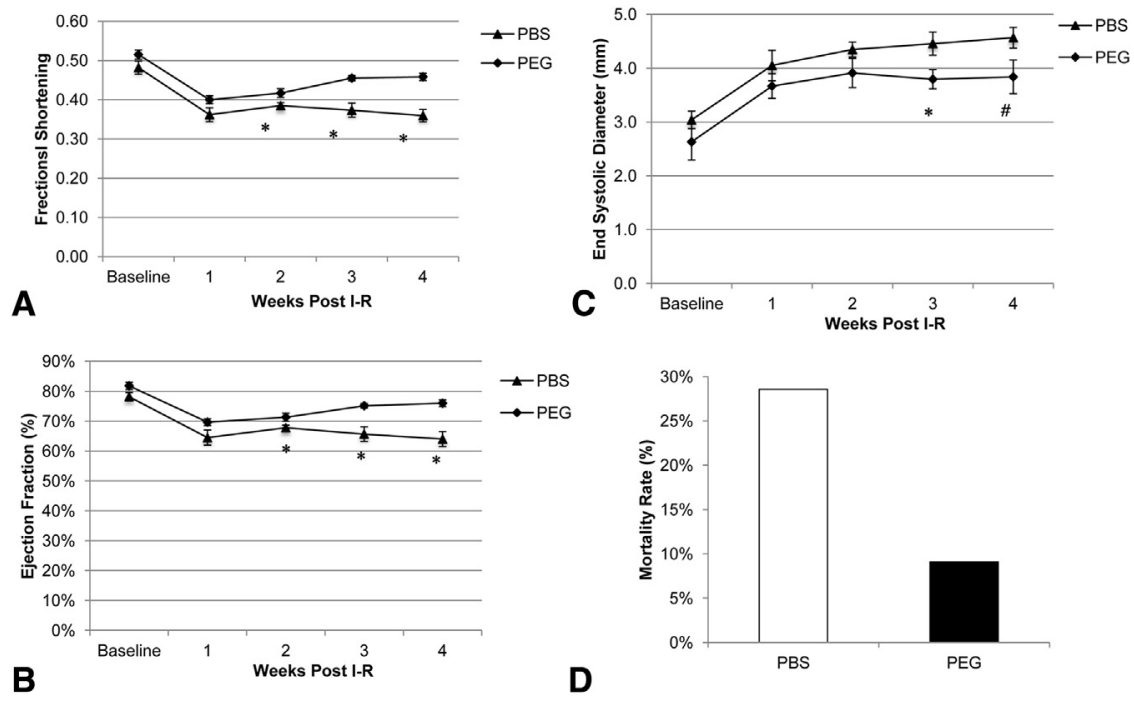

FIGURE 2. PEG treatment improves cardiac function following I-R injury. Cardiac function was studied by echocardiography in rats 4 weeks following I-R injury and treatment with either intravenous PBS or PEG. A, Fractional shortening is significantly higher in the PEG compared to the PBS group at 2 to 4 weeks. $\mathrm{n}=6$ in each group; $* P<.03$ versus PBS. B, Ejection fraction in PBS- versus PEG-treated rats up to 4 weeks post I-R injury. $\mathrm{n}=6$ in each group; ${ }^{*} P<.04$ versus PBS. C, End-systolic diameter is significantly smaller in PEG versus PBS groups at 3 and 4 weeks post I-R injury. $\mathrm{n}=6$ in each group; ${ }^{*} P<.03$ versus PBS, $\# P=.05$ versus PBS. D, Mortality rate at 48 hours following 1 hour ischemia in PBS- and PEG-treated animals. $\mathrm{n}=14$ for PBS and 11 for PEG. $P=.34$ using Fisher exact test. $P E G$, Polyethylene glycol; $P B S$, phosphate-buffered saline; $I-R$, ischemia-reperfusion. 


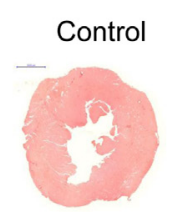

A
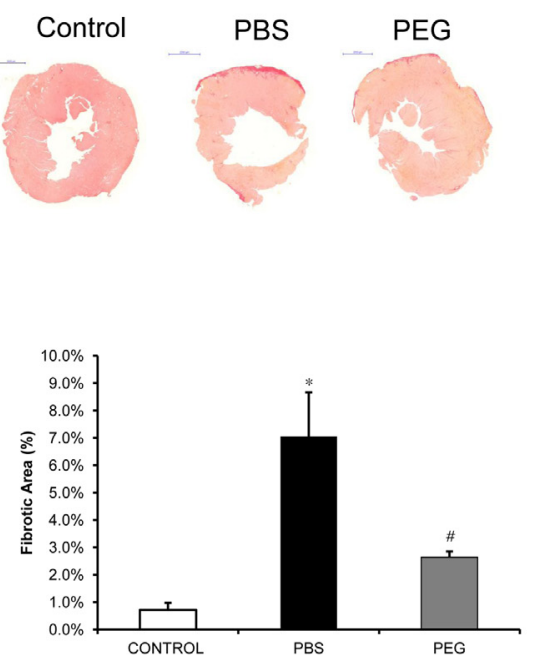

B

FIGURE 3. PEG treatment decreases fibrosis at 4 weeks following I-R injury. A, Representative photomicrographs of Picro-Sirius Red-stained left ventricles of control rats and PBS- or PEG-treated rats harvested 4 weeks post I-R injury. B, Quantitation of fibrotic area (red staining area) of left ventricle. $\mathrm{n}=5$ to 6 per group; $* P=.001$ versus Control, $\# P<.01$ versus PBS. C, Representative photomicrographs of Masson trichrome-stained left ventricles from control, PBS-treated, and PEG-treated rats harvested 4 weeks post-I-R injury. D, Quantitation of left ventricular fibrotic area (purple/blue staining area). $\mathrm{n}=5$ to 6 per group; $* P<.001$ versus Control, $\# P<.001$ versus PBS. $P E G$, Polyethylene glycol; $P B S$, phosphate-buffered saline.

nuclei in the LV at 48 hours after I-R. As seen in Figure $3, A$ and $B$, the number of TUNEL-positive cells was increased 3.4-fold in the PBS group. Treatment with PEG prior to reperfusion led to a significant decrease in apoptosis as there was only a 1.4-fold increase in TUNEL-positive cells (Figure 4, A and $B$ ). To further assess the effects of PEG on apoptotic signaling, caspase 3 activity was studied by measuring expression of cleaved caspase 3. Correlating with the TUNEL results, cleaved caspase 3 expression was more than 2-fold greater in the PBS group compared to the PEG-treated group (Figure 4, $C$ and $D$ ).

\section{Treatment With PEG Upregulates Cell Survival Signaling In Vivo}

PEG treatment led to a significant reduction in ischemiaperfusion-induced myocardial apoptosis and, therefore, we
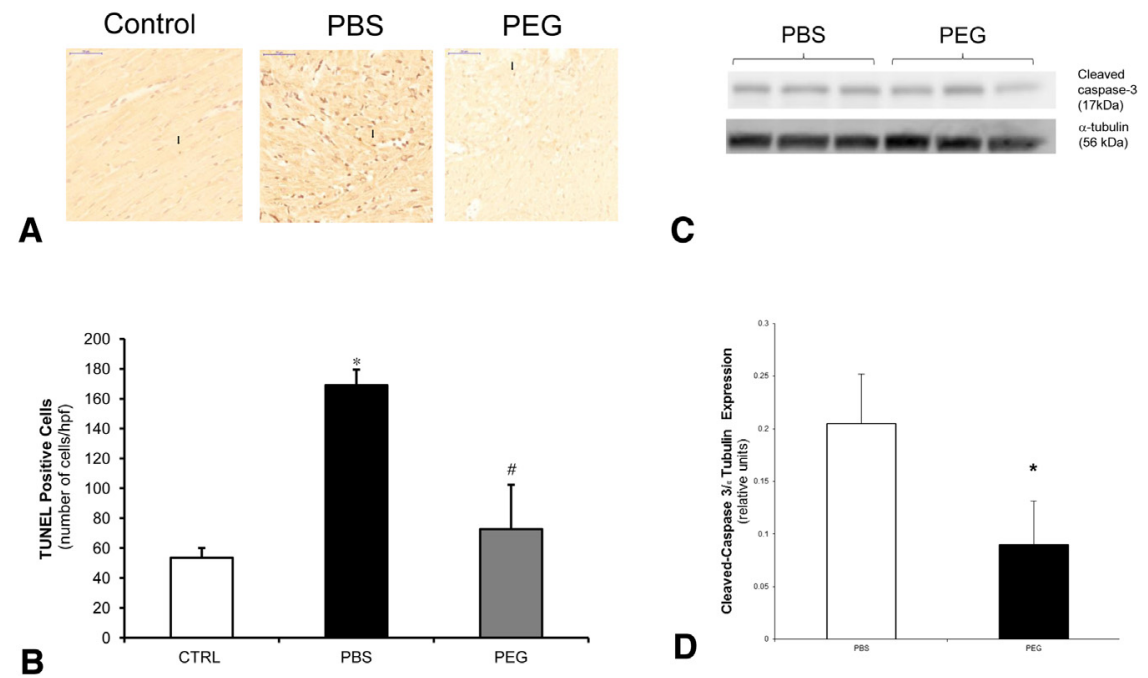

FIGURE 4. PEG treatment decreases apoptosis following I-R injury. A, Representative photomicrographs of TUNEL staining in left ventricles of control rats and PBS- or PEG-treated rats harvested 48 hours post-I-R injury. Black arrows show examples of TUNEL-positive cells in each image. B, Quantitation of TUNEL-positive cells/hpf. $\mathrm{n}=3$ to 5 per group; ${ }^{*} P=.01$ versus Control, $\# P<.02$ versus PBS. C, Representative immunoblot shows cleaved caspase 3 expression in left ventricles at 48 hours following I-R in rats treated with PBS or PEG. $\alpha$-Tubulin was used as a loading control. D, Densitometric analysis of cleaved caspase $3 / \alpha$-tubulin expression. $\mathrm{n}=5$ per group; $* P<.003$ versus PBS. $P B S$, Phosphate-buffered saline; $P E G$, polyethylene glycol; TUNEL, terminal deoxynucleotidyl transferase-mediated dUTP nick-end labeling; $h p f$, high-powered field; $C T R L$, control. 

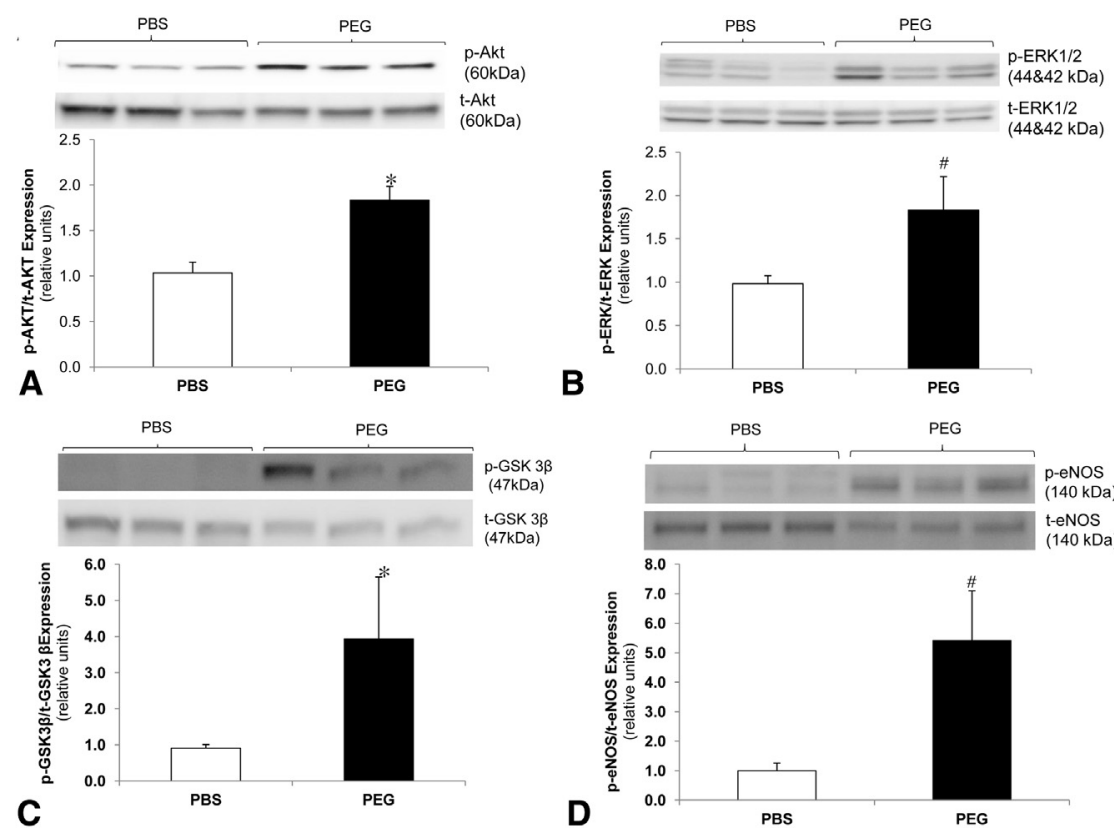

FIGURE 5. Increased left ventricular survival signaling with PEG treatment at 48 hours post-I-R injury. A, Representative immunoblot shows phosphorylated Akt $(p-A k t)$ and total Akt $(t-A k t)$ expression in left ventricles at 48 hours following I-R in rats treated with PBS or PEG and densitometric analysis of pAkt/t-Akt expression. $\mathrm{n}=4$ to 5 per group; $* P<.002$ versus PBS. B, Representative immunoblot of phosphorylated ERK1/2 ( $p$-ERK1/2) and total ERK1/2 $(t$-ERK1/2) expression and densitometric analysis of p-ERK1/2:t-ERK1/2 expression. $\mathrm{n}=4$ per group; $\# P<.05$ versus PBS. C, Representative immunoblot shows phosphorylated GSK $3 \beta$ ( $p$-GSK $3 \beta)$ and total GSK $3 \beta(t-G S K 3 \beta)$ expression and densitometric analysis of $\mathrm{p}$-GSK $3 \beta / \mathrm{t}-\mathrm{GSK} 3 \beta$ expression. $\mathrm{n}=4$ to 5 per group; $* P<.05$ versus PBS. D, Representative immunoblot shows phosphorylated eNOS $(p-e N O S)$ and total eNOS $(t-e N O S)$ expression at 48 hours following I-R in rats treated with PBS or PEG and densitometric analysis of $\mathrm{p}$-eNOS/t-eNOS expression. $\mathrm{n}=5$ per group; $\# P<.02$ versus PBS. $P B S$, Phosphate-buffered saline; $P E G$, polyethylene glycol; eNOS, endothelial nitric oxide synthase.

went on to investigate cell survival signaling. Akt (protein kinase B) signaling was increased in the PEG-treated hearts 2-fold versus the PBS controls as measured by Akt phosphorylation (Figure 5, $A$ ). ERK1/2 signaling was upregulated greater than 2-fold (Figure 5, B) and phosphorylation of the prosurvival signaling molecule, GSK- $3 \beta$, was also increased nearly 4-fold in the PEG-treated group versus controls (Figure 5,C). eNOS activity was significantly upregulated in the PEG-treated hearts by approximately 2.5-fold (Figure 5, D), once again demonstrating PEG-mediated activation of cell survival signaling.

\section{DISCUSSION}

This is the first report describing the use of PEG to protect the heart from I-R injury in an in vivo model. In this study, we have shown that the protective effects of PEG 15-20 that were seen in cardiac myocytes subjected to hypoxiareoxygenation injury in vitro ${ }^{1}$ are translatable to an in vivo rat model of I-R injury. Intravenous delivery of $10 \%$ PEG 15-20 prior to reperfusion following 60 minutes of ischemia by LAD occlusion resulted in significant recovery of $\mathrm{LV}$ function, markedly less myocardial apoptosis as measured by TUNEL staining and caspase 3 activity, and inhibition of ventricular fibrosis and adverse remodeling. Similar to our studies using PEG in primary cultures of neonatal rat cardiac myocytes exposed to hypoxia and reoxygenation, PEG treatment again led to the upregulation of myocardial prosurvival signaling as measured by the phosphorylation of Akt, ERK1/2, GSK-3 $\beta$, and eNOS, which was indicative of increased activity of these signaling pathways.

Based on previous in vitro studies, PEG plays an important role in stabilization and preservation of lipid-raft domains leading to maintenance of sarcolemmal membrane integrity and upregulation in cell survival signaling. ${ }^{1,2}$ Lipid-raft coalescence in association with apoptosis is a known sequela of cell injury. ${ }^{4}$ Lipid rafts are sphingolipid and cholesterol-rich domains of the plasma membrane that contain a variety of signaling and transport proteins. There are different subtypes of lipid rafts that can be distinguished based on their protein and lipid composition. Caveolae, a subset of lipid rafts, are flask-like invaginations of plasma membrane that contain proteins of the caveolin family such as caveolin 1,2 , and $3 .^{5}$ The presence within lipid rafts of several membrane proteins involved in cell signaling has led to the consensus that these lipid domains play an important role in the process of signal transduction. ${ }^{6}$ In some cases, preassembled signaling complexes are localized in lipid-raft domains. The phosphatidylinositol3-kinase/protein kinase B (PI3K/PKB, Akt) pathway is a protein kinase system that interacts with caveolin, and this 
interaction may regulate cell survival. ${ }^{7}$ Receptor tyrosine kinases have also been shown to be localized to caveolae, including epidermal growth factor, nerve growth factor, insulin-like growth factor, and platelet-derived growth factor, and their downstream effector mitogen-activated protein kinases, which regulate numerous cellular processes, are also regulated by caveolin. ${ }^{8,9}$ eNOS activity is also regulated by binding to caveolin. ${ }^{10}$ Therefore, the beneficial effects of PEG in the upregulation of prosurvival signaling may be mediated, in large part, by preservation of lipid-raft domains.

PEGs are multifunctional molecules that can target several pathologic processes associated with organ injury following I-R, including tissue edema, cell membrane dysfunction, immune activation, and tight junction function. ${ }^{11}$ Because of their large molecular size and hydrophilic properties, PEGs generate an oncotic sink to reduce cellular and tissue edema. PEG can attenuate lipid peroxidation and cellular membrane injury. Previous studies have demonstrated the efficacy of PEG 15-20 in protecting intestinal epithelial cells against various injurious stimuli including radiation injury ${ }^{2}$ and bile acid exposure. $^{12}$

PEG has been used both experimentally and clinically for myocardial preservation in the transplantation setting. The only previously reported clinical study utilized standard cardioplegia solution with 5\% PEG 20 during the donor heart procurement in 22 patients. ${ }^{13}$ This was associated with a significant decline in acute rejection episodes during the first year after transplant compared with standard cardioplegia solution. Although there were no mechanistic studies performed, it was hypothesized that there may have been less preservation injury with the addition of PEG. PEG compounds have also been described to be used in early clinical trials for renal $^{14}$ and liver transplantation. ${ }^{15,16}$ PEGs, particularly those with low molecular weights, are considered to be relatively nontoxic; however, the potential side effects of PEG 15-20 when delivered intravenously in humans are unknown and toxicity studies would need to be conducted.

In conclusion, our results suggest that PEG 15-20 can be safely delivered intravenously and is very effective in minimizing normothermic myocardial I-R injury through the inhibition of apoptotic signaling and upregulation of cell survival signaling, leading to less ventricular remodeling and preservation of ventricular function. As there are currently limited strategies to inhibit or decrease acute I-R injury in the setting of acute coronary syndrome and emergent nonsurgical revascularization, PEG 15-20 appears to be an intriguing polymer-based therapeutic approach for this important clinical problem. In addition, PEG may prove to be beneficial in cardiac surgery, potentially by being added to cardioplegia solution for complex operations requiring long aortic cross-clamp times and for patients with severe ventricular dysfunction or in the setting of cardiac transplantation as a component of the organ preservation solution.

\section{References}

1. Malhotra R, Valuckaite V, Staron ML, Theccanat T, D'Souza KM, Alverdy JC, et al. High molecular weight polyethylene glycol protects cardiac myocytes from hypoxia and reoxygenation-induced cell death and preserves ventricular function. Am J Physiol Heart Circ Physiol. 2011;300:H1733-42.

2. Valuckaite V, Zaborina O, Long J, Hauer-Jensen M, Wang J, Holbrook C, et al Oral PEG 15-20 protects the intestine against radiation: role of lipid rafts. Am J Physiol Gastrointest Liver Physiol. 2009;297:G1041-52.

3. Rubio-Gayosso I, Platts SH, Duling BR. Reactive oxygen species mediate modification of glycocalyx during ischemia-reperfusion injury. Am J Physiol Heart Circ Physiol. 2006;290:H2247-56.

4. Bionda C, Hadchity E, Alphonse G, Chapet O, Rousson R, RodriguezLafrasse C, et al. Radioresistance of human carcinoma cells is correlated to a defect in raft membrane clustering. Free Radic Biol Med. 2007; 43:681-94.

5. Pike LJ. Lipid rafts: bringing order to chaos. J Lipid Res. 2003;44:655-67.

6. Michel V, Bakovic M. Lipid rafts in health and disease. Biol Cell. 2007;99: 129-40.

7. Zhuang L, Lin J, Lu ML, Solomon KR, Freeman MR. Cholesterol-rich lipid raft mediate Akt-regulated survival in prostate cancer cells. Cancer Res. 2002;62: 2227-31.

8. Das M, Das DK. Lipid raft in cardiac health and disease. Curr Caridol Rev. 2009; 5:105-11.

9. Couet J, Sargiacomo M, Lisanti MP. Interaction of a receptor tyrosine kinase, EGF-R, with caveolins. Caveolin binding negatively regulates tyrosine and serine/threonine kinase activities. J Biol Chem. 1997;272:30429-38.

10. García-Cardeña G, Fan R, Stern DF, Liu J, Sessa WC. Endothelial nitric oxide synthase is regulated by tyrosine phosphorylation and interacts with caveolin1. J Biol Chem. 1996;271:27237-40.

11. Valuckaite V, Seal J, Zaborina O, Tretiakova M, Testa G, Alverdy JC. High molecular weight polyethylene glycol (PEG 15-20) maintains mucosal microbial barrier function during intestinal graft preservation. J Surg Res. 2013;183: 869-75.

12. Oltean M, Joshi M, Herlenius G, Olausson M. Improved intestinal preservation using an intraluminal macromolecular solution: evidence from a rat model Transplantation. 2010;89:285-90.

13. Collins GM, Wicomb WN, Levin BS, Verma S, Avery J, Hill JD. Heart preservation solution containing polyethylene glycol: an immunosuppressive effect? Lancet. 1991;338:890-1.

14. Hauet T, Goujon JM, Baumert H, Petit I, Carretier M, Eugene M, et al. Polyethylene glycol reduces the inflammatory injury due to cold ischemia/reperfusion in autotransplanted pig kidneys. Kidney Int. 2002;62:654-67.

15. Franco-Gou R, Mosbah IB, Serafin A, Abdennebi HB, Rosello-Catafau J, Peralta C. New preservation strategies for protecting liver grafts against cold ischemia reperfusion injury. J Gastroenterol Hepatol. 2007;22:1120-6.

16. Abbas R, Kombu RS, Dignam D, Gunning W, Stulberg JJ, Brunengraber H, et al. Polyethylene glycol modified-albumin enhances the cold preservation properties of University of Wisconsin solution in rat liver and a hepatocyte cell line. J Surg Res. 2010;164:95-104. 